\section{Evaluating the relationship between smash scores and jump parameters of elite level badminton players}

\section{Elit badmintoncuların smaç vuruşu isabet oranları ve sıçrama performansları ilişkisinin değerlendirilmesi}

\section{Abstract}

The aim of this study was to evaluate the relationship between the strength and jump parameters of elite level badminton players in the range oscoref 5, 10, and 15 intervals of smash. A total of 10 male national badminton players with a mean age of $15.2 \pm 0.18$ years, length of $170.5 \pm 2.13 \mathrm{~cm}$, body weight of 59.5 $\pm 2.48 \mathrm{~kg}$ and sports age of $5 \pm 1.36$ years participated in the study. Leg strength test dynamometer (Prosport-TMR HBD 1000 dynamometer), jump parameters of the subjects were measured with Opto Jump (Microgate, Bolzano). The smash swere performed as $3 \times 30$ sec and 3 minutes passive rest protocol was applied after each series. In every 30 seconds, the shots were made by the national team coach with the multishuttle method as 5-10-15. The hit of the parallel smash scored was recorded by the referee. Data analysis was performed using SPSS 23.0 package program. For the relationship between the parameters, Spearman Correlation test and Friedman test were used for comparison of parameters with intermittent jump. As a result of the study, it was seen that there were statistically significant differences in the power and contact time parameters applied

\author{
Özlem Orhan ${ }^{1}$ \\ Ebru Çetin ${ }^{2}$ \\ İmdat Yarım ${ }^{3}$ \\ Okan Gültekin ${ }^{4}$
}

\section{Özet}

Bu çalışma elit seviyedeki badmintoncuların 30 sn içinde yaptukları 5,10 ve 15 aralıklı smaç vuruşu atış performanslarının kuvvet ve sıçrama parametreleri ile ilişkilerinin değerlendirilmesi amaciyla yapılmıştur. Çalışmaya Ankara Büyükşehir Belediyesi ve Ego Spor Kulübü yaş ortalamas1 15.2 \pm 0.18 yil, boy uzunluğu $170.5 \pm 2.13 \mathrm{~cm}$, vücut ağırlığ1 $59.5 \pm 2.48 \mathrm{~kg}$ ve spor yaş1 $5 \pm 1.36$ yll olan toplam 10 erkek milli badmintoncu katılmıştır. Araştırmaya katılan deneklerin bacak kuvveti testi dinamometre (Prosport- TMR HBD 1000 dinamometre), sıçrama yükseklikleri ve havada kalış süreleri Opto Jump (Microgate, Bolzano) cihazı ile ölçülmüştür. Smaç vuruşları 3x30 sn şeklinde yapılmış ve her seri sonrası $3 \mathrm{dk}$ pasif dinlenme protokolü uygulanmıştır. Her bir 30 sn 'de atışlar 5-10-15 şeklinde milli takım antrenörü tarafindan top besleme yöntemi ile yapılmıştur. Paralele atılan smaç vuruşlarının isabeti ise hakem tarafindan kaydedilmiştir. Verilerin analizi SPSS 23.0 paket programında yapılmıştır. Parametreler arasında ilişki için, Spearman Korelasyon test ve aralıklı sıçrama ile parametrelerin karşılaştırlmasında Friedman test uyguland. İstatiksel olarak anlamlllik düzeyi

\footnotetext{
${ }^{1}$ Doç. Dr., Gazi Üniversitesi, Spor Bilimleri Fakültesi, Antrenörlük Eğitimi, dr.ozlemorhan@gmail.com

${ }^{2}$ Doç. Dr.,Gazi Üniversitesi, Spor Bilimleri Fakültesi, Beden Eğitimi ve Spor Öğretmenliği, cetinebruu@gmail.com

${ }^{3}$ Doç. Dr., Gazi Üniversitesi, Spor Bilimleri Fakültesi, Beden Eğitimi ve Spor Öğretmenliği, imdat@gazi.edu.tr

${ }^{4}$ Gazi Üniversitesi, Spor Bilimleri Fakültesi, Antrenörlük Eğitimi okangultekin13@gmail.com
} 
Orhan, Ö., Çetin, E., Yarım, İ., \& Gültekin, O. (2019). Elit badmintoncuların smaç vuruşu isabet oranları ve sıçrama performansları ilişkisinin değerlendirilmesi. Journal of Human Sciences, 16(3), 815-822. doi:10.14687/jhs.v16i3.5761

during the jumps as the number of smash increased within 30 seconds. In addition, it was found that as the frequency of scored increased, the rate of accuracy and heart rate (KAS) increased. This can be interpreted as a decrease in the scored of the players due to fatigue as the smash frequency increases.

Keywords: Badminton, Jump, Smash.

(Extended English summary is at the end of this document) $\mathrm{p}<0.05$ olarak kabul edilmiștir. Araștırmanın sonucunda 30 sn içinde yapılan atış sayısı arttıkça sıçramalar sırasında uygulanan power ve contact time parametrelerinde istatistiksel olarak anlamlı farkllilklar olduğu görülmüştür. Bunun yanı sıra atış sıklığ1 arttıkça isabet oranlarında azalma ve kalp atım sayısında (KAS) artış olduğu tespit edilmiştir. $\mathrm{Bu}$ durum atış sıklığ 1 arttıkça oluşan yorgunluk sebebiyle, oyuncuların isabet oranlarında bir azalmaya sebep olabileceği şeklinde yorumlanabilir.

Anahtar Kelimeler: Badminton, Smaç Vuruşu, Siçrama.

\section{Giriş}

Badminton, iki veya dört oyuncunun topu yere düşürmeden, raketle karşllkklı vuruş esasına dayalı, file üstünde oynanan olimpik bir spordur (Gülmez, 2007). Bu spor dalı tekrarlayan yüksek şiddetli oyunların (rally) kısa dinlenme araları ile kesildiği, yüksek düzeyde aerobik ve anaerobik güç özelliklerinin önemli olduğu düzensiz aralıklı bir spor aktivitesi olarak tanımlanmıştır (Faccini \& Dal Monte, 1996; Cabello Manrique \& Gonzalez-Badillo, 2003; Faude ve ark., 2007). Badminton maçının yapısı incelendiğinde ise kısa dinlenme süreleriyle birlikte kısa süreli ve yüksek yoğunluklu hareketlerle karakterize olduğu, hızlı yön değişiklikleri, sıçramalar, kesintisiz hamleler, hızlı kol hareketleri ve çeşitli kısa ve patlayıcı hareketler gerektirdiği bilinmektedir (Omesegaard, 1996). Oyunun karakteristik özelliği dikkate alındığında başarılı bir oyuncu için sürat, dayanıklılık, kuvvet, koordinasyon, reaksiyon, sezinleme, mental rotasyon, denge ve teknik özellikler ön plana çıkmaktadır.

Smaç vuruşu badmintonda sayı için önemli vuruşlardandır ve gücün ise vuruşun temelini oluşturduğu düşünülmektedir (El-Gizawy, 2014). Lo ve Stark 'a (1991), göre smaç vuruşunda en etkili unsurların güç ve hız olduğuna dikkat çekilmiştir. Raman ve arkadaşları ise kazanılmak istenen bir badminton oyunun yapısında sporcuların sürat, çeviklik, dayanıklılık, esneklik ve kuvvet gibi motorik özellikleri en yüksek limitlerinde ve kısa sürede uygulamaları gerektiğini belirtmişlerdir (Raman \& Nageswaran, 2013). Yapılan bir başka çalışmada ise badminton oyuncuların motorik özellikleri, kuvvet ve kassal dayanıklılık arasında anlamlı bir ilişki olduğu bulunmuştur (Tiwari, Rai \& Srinet, 2011). Jaworski ve Zak (2016) ise 11-19 yaş grubu badmintoncular üzerinde yaptıkları çalışmada çeviklik, esneklik, bacak kuvveti ve dayanıklılığın oyun kalitesini ve sonucunu etkileyen değişkenler olabileceğini belirtmiştir.

Oyun akışı içerisinde uygulanan atış ve vuruşlar arasında smaç vuruşu, çoğunlukla bitirici atış olarak tanımlanır. Sayı kazanılan atışların dağılımı incelendiğinde \% 53,9 oranında smaç vuruşları öne çıkmaktadır(Tong \& Hong, 2000). Smaç vuruşunda sıçrama badmintonda kullanılan popüler bir ölçektir. Başarılyla gerçekleştirildiğinde hem puan açısından hem de aldatma becerisinin etkili uygulanması için önemlidir (Sakurai \& Ohtsuki, 2000). Smaç vuruşunda diğer önemli bir faktör ise bacak kuvvetidir. Badmintoncuların bacak kuvveti ile smaç vuruşunun incelendiği çalışmalarda bacak kuvveti ile smaç vuruş performansının etkili olabileceği düşünülür (Sakurai \& Ohtsuki, 2000; Tsai ve ark., 2006). Badmintoncuların smaç vuruşunu etkileyen faktörlerin incelendiği bir çalışmada kuvvet ile smaç vuruşu arasındaki pozitif ilişkiye dikkat çekilmiş ve ayrıca hızın, tekniğin smaç vuruşunun kalitesini belirleyen önemli faktörler olduğu belirtilmiştir. Sıçrama performansının yanı sıra bu teknikte üst gövdenin katılımı ve gövdeyi döndürme kuvvetinin de atış kalitesini etkileyebileceği belirtilmiştir (Hirashima \& ark., 2002; Kimura \& ark., 2014). 
Orhan, Ö., Çetin, E., Yarım, İ., \& Gültekin, O. (2019). Elit badmintoncuların smaç vuruşu isabet oranları ve sıçrama performansları ilişkisinin değerlendirilmesi. Journal of Human Sciences, 16(3), 815-822. doi:10.14687/jhs.v16i3.5761

Badminton müsabakalarında oyun kazanmak için etkili faktörler dikkate alındığında pek çok becerinin komplike kullanıllyor olabilmesi dikkat çekmektedir. Oyunun akıcı olmasi, hızlı hareket etme, yer-yön değiştirme becerisi özellikle sayı kazanmada etkili faktörler arasındadır (Raman \& Nageswaran, 2013). Sadece güçlü yada hızlı olmak mı yoksa güç ve hızı doğru zamanda kullanabilmek mi ? Tüm bunların yanı sıra topa etkili ve isabetli vuruşlar uygulayabilmek için kortta doğru ve erken pozisyon almak mi esastrr? Bu ayrıntılar değerlendirildiğinde oyunda en iyi sonucu elde etmenin, saha içerisinde hızlı ve çabuk bir şekilde hareket etme yeteneğine bağlı olduğu düşünülmektedir (Mahoney\&Sharp, 1995). Yapılan araşturmalar ve elde edilen bilgiler incelendiğinde isabet oranları açısından etkili unsurların hangileri olduğunun belirlenebildiği herhangi bir çalışmaya rastlanamamıştır. Bu amaçla elit seviyedeki badmintoncuların 30 sn içinde yaptıkları 5,10 ve 15 aralıklı smaç vuruşu atış performansları sırasındaki sıçrama becerileri, isabet oranları ve KAS açılardan değerlendirilmiştir. Bunun yanı sıra paralele yapılan smaç atışları sırasında kaydedilen isabet oranları, sıçrama performansları ve sürat parametreleri bacak kuvveti ile ilişkilendirilmiştir.

\section{Yöntem}

Çalışmaya Ankara Büyükşsehir Belediyesi ve Ego Spor Kulübü yaş ortalamas1 15.2£0.18 y1l, boy uzunluğu $170.5 \pm 2.13 \mathrm{~cm}$, vücut ağırllğı $59.5 \pm 2.48 \mathrm{~kg}$ ve spor yaş1 $5 \pm 1.36$ yıl olan toplam 10 erkek milli badmintoncu katıldı. Katılımcılara gönüllü onam formu imzalatıldı ve çalışma Helsinki Deklarasyonu İlkelerince yürütüldü. Çalışma iki gün olacak şekilde planlandı ve ilk gün boy, vücut ağırlığ1 (Seca) ve bacak kuvveti (Prosport- TMR HBD 1000 dinamometre) ölçüldü. İkinci gün ise Squat Jump (SJ) sıçraması kaydedildikten sonra smaç vuruşu protokolü uygulandi; sıçrama yükseklikleri ve havada kalış süreleri Opto Jump (Microgate, Bolzano) cihazı ile ölçüldü. Cihaz oyuncuların paralel smaç vuruşu yaptıkları alana (şekil 1) yerleştirildi. Tüm sıçramaların dataları kaydedildi.

\section{Paralel smaç vuruşu}

Paralel smaç vuruşu $3 \times 30$ sn şeklinde yapılmış ve her seri sonrası $3 \mathrm{dk}$ pasif dinlenme protokolü uygulanmıştır. Her bir 30 sn 'de atışlar 5-10-15 şeklinde milli takım antrenörü tarafindan top besleme yöntemi ile yapılmıştır. Paralele atılan smaç vuruşlarının isabeti ise hakem tarafindan kaydedilmiştir.

Smaç vuruşları için standart badminton sahası (şekil 2) kullanıldı. Badminton sahası içerisinde dip kort çizgisi ile orta kort arasında belirlediğimiz alandan (şekil 1) oyunculara paralel smaç vuruşu yaptırıldı ve tarafimızca belirlenmiş olan 80x100 cm dikdörtgen şeklindeki (fileye 2.60 $\mathrm{cm}$, arka dip kort çizgisine $1.28 \mathrm{~cm}$ ve merkez çizgisine $1.50 \mathrm{~cm}$ uzaklıkta) alana deneklerin attıkları topların isabet oranları kaydedildi.

Yapılan smaç vuruşları milli takım antrenörü tarafindan top besleme yöntemi kullanılarak yapılmıştır. 3x30 sn şeklinde yapılan atışlarda her bir 30 sn arasında $3 \mathrm{dk}$ dinlenme uygulanmıştır. Atışlarda sporcu filenin karşısından dip korttan arka kort bölgesine paralel smaç vuruşu yapmıştır. 
Orhan, Ö., Çetin, E., Yarım, İ., \& Gültekin, O. (2019). Elit badmintoncuların smaç vuruşu isabet oranları ve sıçrama performansları ilişkisinin değerlendirilmesi. Journal of Human Sciences, 16(3), 815-822. doi:10.14687/jhs.v16i3.5761

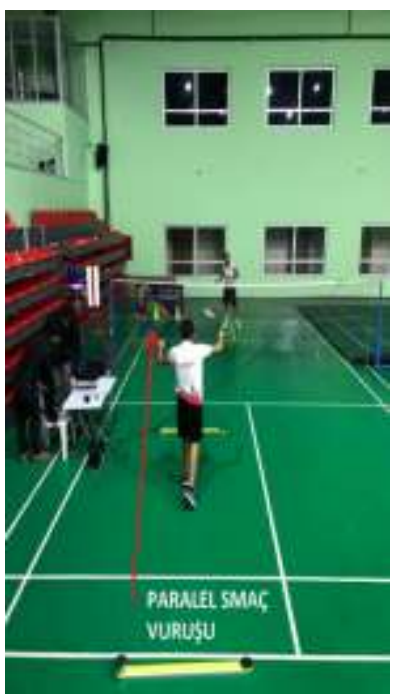

Şekil 1: Opto jump cihazı yerleşimi
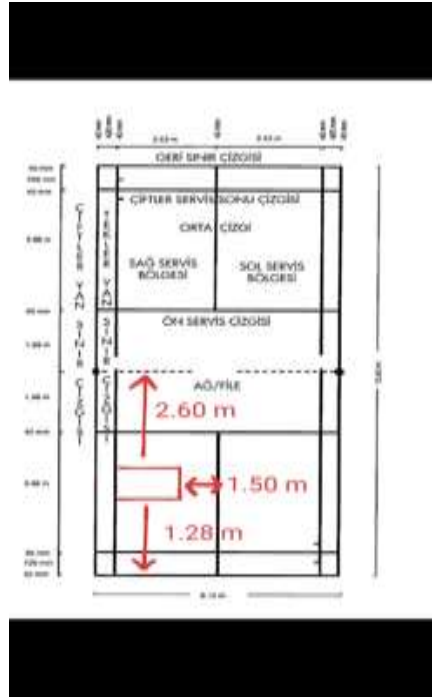

Şekil 2: Badminton sahası ve isabet alanı

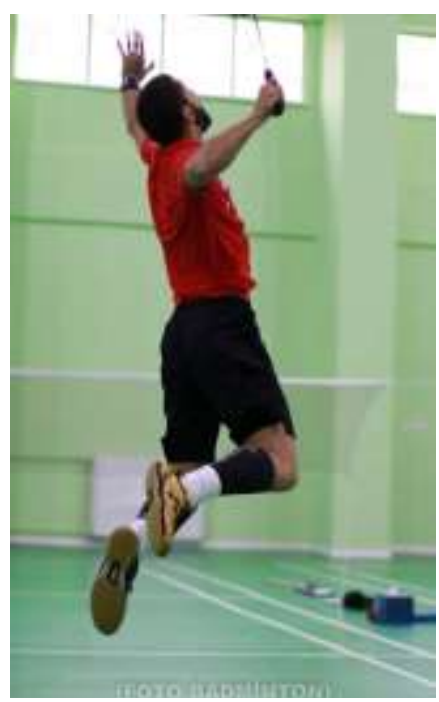

Şekil 3: Smaç vuruşu

İstatiksel Analiz: Verilerin analizi SPSS 23.0 paket programında yapıldı. Tanımlayıcı istatistikler yapıldıktan sonra parametreler arasında ilişki için, Spearman Korelasyon test ve aralıklı sıçrama ile parametrelerin karşılaştırılmasında ise Friedman uygulandı. İstatiksel olarak anlamlılık düzeyi $\mathrm{p}<0.05$ olarak kabul edilmiştir.

\section{Bulgular}

30 saniye içinde 5, 10, 15 atış şeklinde yapılan aralıklı smaç sıçrama performanslarındaki bulgular değerlendirilmiştir. Atış performansları sırasında kaydedilen sıçrama parametreleri değerlendirildiğinde atış sayıları dikkate alınarak yapılan karşılaştırmada contact time ve power sonuçlarının 5-15 atış arasında istatistiksel olarak farklı olduğu tespit edilmiştir. Sıçrama yükseklikleri ve T-Flight parametrelerinde ise anlamlı herhangi bir farklılığa rastlanmamıştur.

İsabet oranları incelendiğinde oyuncular 5 atışta \%36, 10 atışta \%26 ve 15 atışta \%23 oranında isabet kaydettiler. KAS incelenmesinde de atış sürelerinin sonunda 5 atışta $95.9 \pm 2.25$ atım/dk, 10 atışta $152.8 \pm 3.5 \mathrm{atım} / \mathrm{dk}$ ve 15 atışta $166.4 \pm 1.34 \mathrm{atım} / \mathrm{dk}$ değerleri ölçüldü. Kaydedilen isabet oranları ile smaç vuruşu sıçrama yükseklikleri bacak kuvveti ve 15 atış isabet oranlarının ilişkilerinin değerlendirilmesi sonucu istatistiksel olarak anlamlı herhangi bir sonuç elde edilememiştir.

Tablo 1: 30 sn içerisinde yapılan farklı sayıdaki atışlarda elde edilen sıçrama performanslarının karşılaştırılması

\begin{tabular}{|c|c|c|c|c|c|c|}
\hline Parametre & $\begin{array}{c}\text { Zaman } \\
\text { (30 sn/atıs sayısı) }\end{array}$ & Ort. \pm SS & $\chi^{2}$ & df & $p$ & Farklar \\
\hline \multirow{3}{*}{ Contact time } & 5 & $3.76 \pm 1.37$ & \multirow{3}{*}{9.8} & \multirow{3}{*}{2} & \multirow{3}{*}{$0.01 *$} & \multirow{3}{*}{$5-15$} \\
\hline & 10 & $3.03 \pm 0.73$ & & & & \\
\hline & 15 & $2.46 \pm 0.9$ & & & & \\
\hline \multirow{3}{*}{ T- flight } & 5 & $0.62 \pm 0.32$ & \multirow{3}{*}{1.8} & \multirow{3}{*}{2} & \multirow{3}{*}{0.41} & \multirow{3}{*}{ - } \\
\hline & 10 & $0.66 \pm 0.36$ & & & & \\
\hline & 15 & $0.97 \pm 0.81$ & & & & \\
\hline \multirow{3}{*}{ Jump Height } & 5 & $23.7 \pm 7.26$ & \multirow{3}{*}{1.4} & \multirow{3}{*}{2} & \multirow{3}{*}{0.49} & \multirow{3}{*}{ - } \\
\hline & 10 & $21.9 \pm 6.65$ & & & & \\
\hline & 15 & $30.1 \pm 13.6$ & & & & \\
\hline \multirow{3}{*}{ Power } & 5 & $21.6 \pm 12.3$ & \multirow{3}{*}{5.4} & \multirow{3}{*}{2} & \multirow{3}{*}{$0.04 *$} & \multirow{3}{*}{$5-15$} \\
\hline & 10 & $23.9 \pm 10.3$ & & & & \\
\hline & 15 & $24.6 \pm 10.5$ & & & & \\
\hline
\end{tabular}


Orhan, Ö., Çetin, E., Yarım, İ., \& Gültekin, O. (2019). Elit badmintoncuların smaç vuruşu isabet oranları ve sıçrama performansları ilişkisinin değerlendirilmesi. Journal of Human Sciences, 16(3), 815-822. doi:10.14687/jhs.v16i3.5761

Tablo 2: 30 sn içinde yapılan 5, 10, 15 atı̧̧ performansının isabet oranları ve Kalp atım sayısı (KAS) değerleri

\begin{tabular}{|c|c|c|c|}
\hline Parametreler & $\begin{array}{c}\text { İsabet say1s1 } \\
\text { Ort. } \pm \text { SS }\end{array}$ & $\begin{array}{c}\text { İsabet oranları } \\
\% \\
\end{array}$ & $\begin{array}{c}\text { KAS } \\
\text { Ort. } \pm S S\end{array}$ \\
\hline 5 Atış & $1.8 \pm 0.33$ & 36 & $95.9 \pm 2.25$ \\
\hline 10 Atış & $2.6 \pm 0.52$ & 26 & $152.8 \pm 3.5$ \\
\hline 15 Attş & $3.4 \pm 0.76$ & 23 & $166.4 \pm 1.34$ \\
\hline
\end{tabular}

Tablo 3: Oyuncuların bacak kuvveti ile sıçrama, sürat ve isabet oranları parametrelerinin ilişkisi

\begin{tabular}{|c|c|c|c|c|}
\hline Parametreler & Ort. \pm SS & $\begin{array}{c}\text { Bacak Kuvveti (kg) } \\
\text { Ort. } \pm \text { SS }\end{array}$ & $\mathrm{r}$ & $p$ \\
\hline Sürat & $5.53 \pm 0.09$ & & -0.21 & 0.56 \\
\hline $\begin{array}{c}\text { Squat jump } \\
\text { siçrama } \\
\text { yüksekliği }(\mathrm{cm})\end{array}$ & $30.1 \pm 4.11$ & $102 \pm 3.65$ & 0.33 & 0.35 \\
\hline $\begin{array}{c}15 \text { Atış isabet } \\
\text { oranı }\end{array}$ & $5.8 \pm 0.71$ & & 0.29 & 0.41 \\
\hline
\end{tabular}

\section{Tartı̧̧ma ve Sonuç}

Araştırmada elde edilen bulgular doğrultusunda 30 sn içinde yapılan farklı sayıda atış performansları değerlendirildiğinde atış sayısı sıklaştıkça yorgunluğun etkisiyle isabet oranlarında düşme olduğu açıkça görülmektedir. Atış sırasında kaydedilen sıçrama parametrelerine bakıldığında ise atış sayısı arttıkça contact time'ın azaldığ1 ve power'ın arttığ1 tespit edilmiştir. Bu doğrultuda da kalp atım sayısı değerlerine göre yorgunluk oluştuğu görülmüştür. Bacak kuvveti ile sürat, squat jump sıçrama yüksekliği ve isabet oranlarının ilisskilendirilmesinde ise herhangi anlamlı bir ilisski tespit edilememiştir.

Badminton oyuncularının motor becerilerinin incelenip değerlendirildiği pek çok çalışmada smaç atış tekniği yaparken iyi sıçramak iyi performans için en popüler kriter olarak dile getirilmektedir. Sıçrama bütün spor dallarında önemlidir ancak badmintonda oyun içindeki pek çok teknik için başarıyı etkileyen önemli parametreler arasında yer almaktadır (Azmin ve ark., 2005). Sadece smaç vuruş tekniği açısından değil oyun içinde sıkça kullanılan aldatma becerilerinde de kullanılan farklı pek çok tekniğin başarısında da etkilidir. Shetty, yaptığı bir çalışmada motor becerilerin birbirleri ile ilişkisini incelemiştir. Elde ettiği bulgulara göre sıçrama ve alt ekstremite kuvvetinin ilişkili olduğunu belirtmiştir (Shetty, 2002). Badmintonda alt ekstremite kuvveti ve sıçrama ile ilgili çalışmalar yapılmış ancak smaç vuruşu isabet oranı ile ilgili olarak yapılan herhangi bir çalışmaya rastlanılmamıştır (Hughes \& Bopf, 2005; Hughes \& Cosgrove, 2007; Chin ve ark., 1995; Cabello ve ark., 2004).

Araştırmada ise badminton oyuncularının bacak kuvveti ile sürat sıçrama ve isabet oranları ilişkisi değerlendirildiğinde istatistiksel olarak anlamlı herhangi bir sonuca rastlanamamıştır. Ancak sıçrama sıklığı arttıkça oyuncuların sıçrama performanslanına etkisi incelenmiş ve contact time'ın azalması oyuncuların daha fazla güç kullanmasına sebep olduğu düşünülmüştür. Atışlar sırasında kaydedilen KAS parametreleri ile değerlendirme yapıldığında ise belirgin bir yorgunluk oluştuğu görülmektedir. $\mathrm{Bu}$ yorgunluğunda isabet oranlarını negatif yönde etkilemiş olabileceği düşünülmektedir. Badminton oyuncularının dayanıklılık parametresi üzerine Jaworski ve Zak, (2016) bir çalışma yapmışlardır. Üç farklı seviyede müsabık olan 11-19 yaşları arasındaki 26 erkek oyuncunun dayanıkl1lık, esneklik, çeviklik, alt gövde kuvveti ve statik kuvvet gibi motorik becerileri değerlendirmişlerdir (Jaworski \& Zak, 2016). Elde ettikleri sonuçlara göre dayanıklılık 
Orhan, Ö., Çetin, E., Yarım, İ., \& Gültekin, O. (2019). Elit badmintoncuların smaç vuruşu isabet oranları ve sıçrama performansları ilişkisinin değerlendirilmesi. Journal of Human Sciences, 16(3), 815-822. doi:10.14687/jhs.v16i3.5761

performansının oyunun kalitesini baskın olarak etkileyen değişkenlerin başında geldiğini ileri sürmüşlerdir. Yapılan farklı çalışmalarda ise badmintonda reaksiyon ve hareket zamanı iki önemli nöromotor değişken olduğundan da bahsedilmektedir. Hareket zamanı, hareketin başlangıcı (contact time) badmintonun hızı oyun temposu açısından oldukça önemlidir (Nagasawa ve ark., 2012). Oyun içerisinde sürekli değişen bir tempo, karmaşık hareketler sebebiyle motorik parametrelerin yüksek seviyede olması önemlidir. Bu parametreler taktik düşünme, doğrudan öngörme yeteneği ile bağlantılıdır (El-Gizawy, 2011). Oyuncunun, rakip cevaplarını önceden tahmin edebilme yeteneği, farklı oyun pozisyonlarında oyuncu için doğru cevabı temel alan en önemli yaratıc1 kapasitelerdendir. Bu durum, oyuncunun hedefin yanı sıra, hedef tarafindan amaçlanan hedefi tanıma ve yerine getirmesine yardımcı olur (El-Gizawy, 2011; Grice, T., 2008). Araştırmada en çarpıcı sonuç olarak oyuncunun sıçrama sıklığının artması contact time yani hareket zamanı, hareketin başlangicindaki zamanda azalma olduğudur. Bu azalma oyuncunun daha fazla güç sarf etmesi şeklinde bir cevap oluşturmaktadır. Artan güç uygulamasının da beraberinde yorgunluk oluşturduğu kaydedilen KAS değerlerinden de tespit edilmiştir. İsabet oranlarının ortalaması değerlendirildiğinde yüzdelik olarak bakıldığında 30 sn içinde atış sıklığı arttıkça isabet oranında da belirgin bir düşüş olduğu söylenebilir. Oyuncular 5 atışta $\% 36,10$ atışta \%26 ve 15 atışta \%23 oranında isabet kaydettiler. Bu atış performanslarının sergilendiği sürelerin sonunda kaydedilen KAS 5 atışta $95.9 \pm 2.2 \mathrm{atım} / \mathrm{dk}, 10$ atışta $152.8 \pm 3.5 \mathrm{atım} / \mathrm{dk}$ ve 15 atısta $166.4 \pm 1.34 \mathrm{atım} / \mathrm{dk}$ şeklindedir.

Sonuç olarak elde edilen bulgulara göre badminton oyuncularının oyun içinde sıçrama sıklığının artmasıyla birlikte sıçrama performanslarının etkilenebileceği görülmüştür. Sıklı̆ın artması contact time süresinin kısalmasına ve power değerinin artması şeklinde cevap bulmaktadır. Tüm bu sıçrama değişkenlerinin yanı sıra aynı zamanda KAS hızla artmakta ve isabet oranları da düşmektedir. Özetle sıçrama performanslarının kalitesinin maç skoru üzerinde etki oluşturabileceği söylenebilir.

Yazar Notu: Çalışmanın yapılmasında gerekli desteği sağlayan Türkiye Badminton Federasyonu Başkanı Sayın Murat Özmekik'e ve araştırmaya katılan sporculara teşekkür ederiz.

\section{Kaynaklar}

Cabello Manrique, D., \& Gonzalez-Badillo, J. J. (2003). Analysis of the characteristics of competitive badminton. British Journal of Sports Medicine, 37(1), 62-66.

Cabello, D., Padial, P., Lees, A. \& Rivas, F. (2004). Temporal and Physiological Characteristics of Elite Women's and Men's Singles Badminton. International Journal of Applied Sports Sciences. 16 (2), 1-12.

Chin, M.K., Wong, A.S.K. So, R.C.H., Siu, O., Steiniger, K. \& Lo, D.T.L. (1995). Sport specific fitness testing of elite badminton players. British Journal of Sports Medicine. 29, 153-157.

El-Gizawy H, Akl AR. (2014).Relationship between reaction time and deception type during smash in badminton. J Sport Res, 1, 49-56.

El-Gizawy, H., (2011). Designing ameasure for tactical thinking for some attack performances for the high levels players in badminton, in: Games department. Egypt: Alexandria University.

Faccini, P., \& Dal Monte, A. (1996). Physiological Demands of Badminton Match Play. Am. J. Sports.Med, 24(6), $64-67$.

Faude, O., Meyer, T., Rosenberger, F., Fries, M., Huber, G., \& Kindermann, W. (2007). Physiological Characteristics of Badminton Match Play. Eur. J. Appl. Physiol, 100(4), 479485.

Hughes, M.G. \& Cosgrove, M. (2007). Assessment of elite Badminton players. In: Winter, E., Jones, A.M., Bromley, P.D and Mercer. T.H. (eds), Sport and Exercise Physiology Testing Guidelines, The British Association of Sport and Exercise Sciences Guide: Volume I: Sport Testing. Oxon: Routledge (214 - 219). 
Orhan, Ö., Çetin, E., Yarım, İ., \& Gültekin, O. (2019). Elit badmintoncuların smaç vuruşu isabet oranları ve sıçrama performansları ilişkisinin değerlendirilmesi. Journal of Human Sciences, 16(3), 815-822. doi:10.14687/jhs.v16i3.5761

Hughes, M.G., \& Bopf, G. (2005). Relationships between performance in jump tests and speed tests in elite Badminton players. Journal of Sport Sciences. 23 (2),194 - 195.

Jaworski, J., \& Zak, M. (2016). Identification of determinants of sports skill level in badminton players using the multiple regression model. Human Movement.17 (1), 21-28.

Kimura, Y., Tsuda, E.,Y. Hiraga, S. Maeda, S., Sasaki, E.Sasaki, Y. Fujita, Y. Ishibashi, \& M. Makino, M. (2014). Trunk motionand muscular strength affect knee valgus moment duringsingle-leg landing after overhead stroke in badminton. Br J Sports Med, 48, (7), 620620.

Lo D, Stark K. (1991) Sports performance series: The badminton overhead shot. J Strength Cond Res, 13, 6-15

M. Hirashima, H. Kadota, S. Sakurai, K. Kudo, \& T.Ohtsuki. (2002). Sequential muscle activity and its functional role in the upper extremity and trunk during overarm throwing," J Sports Sci, 20, (4), 301-10.

Mahoney, C. A., \& Sharp, N.C.C. (1995). The Phyciological Profile of Elite Junior Squash Players, In: Reilly, T., Huges, M., Lees, A., Science and Racket Sports, E. \& F. N., Sporn, London.

Nagasawa, M., Y. Hatori, M. Kakuta, T. Hayashi \& Y. Sekine, 2012. Smash motion analysis for Badminton from image in: Proceedings of the IIEEJ Image Electronics and Visual Computing Workshop, Kuching, Malaysia.

Omosegaard, B. (1996). Physical Training for Badminton. (8-21). Denmark: Malling Beck.

Raman, D., \& Nageswaran, A.S. (2013). Effect of gamespecific strength training on selected physiological variables among badminton players. Int J Sci Res. 2(10),1-2.

Rambely, A.Z., Bakar W.A., Safrulsahar, Y. (2005).The analysis of the jumping smash in the game of badminton, 23 International Symposium on Biomechanics in Sports.

Sakurai, S., \& Ohtsuki, T. (2000). Muscle activity and accuracy of performance of the smash stroke in badminton with reference to skill and practice. Journal of Sports Sciences, 18, 901-914.

Shetty, A.B. (2002). Leg Power. Sports Biomechanics, 1 (2), 147-155.

Tiwari L.M., Rai V., \& Srinet S. (2011). Relationship of selected motor fitness components with the performance of badminton player. Asian J Phys Educ Comput Sci Sports, 5 (1), 88-91.

Tong, Y.M. \& Hong, Y. (2000). The playing pattern of world's top single badminton players. in Proceedings of XVIII International Symposium on Biomechanics in Sports (pp.825-830). Hong Kong: The Chinese University of Hong Kong.

Tsai, C. L., Yang, C. C., Lin, M. S., Huang, K. S., \& Chang, S. S. (2006). The EMG activity of the lower extremities in badminton smash stroke. Journal of Biomechanics, 39, (562).

\section{Extended English Summary}

Badminton is an Olympic sport played on a net with two or four players based on mutual rackets without falling the ball to the ground (Gülmez, 2007). This sport is defined as an irregular intermittent sport activity in which repetitive high-intensity games (rally) are interrupted by short resting breaks and high aerobic and anaerobic power properties are important (Faccini \& Dal Monte, 1996; Cabello Manrique \& Gonzalez-Badillo, 2003; Faude et al., 2007). Smash is one of the important shots in badminton and power is thought to be the basis of the shot (El-Gizawy, 2014). Among the shots and shots applied in the game flow, the smash is often defined as a finishing shot. When the distribution of the number of shots is examined, smash stand out at a rate of $53.9 \%$ (Tong \& Hong, 2000). The aim of this study was to evaluate the relationship between the strength and jump parameters of elite level badminton players in the range oscoref 5,10, and 15 intervals of smash. A total of 10 male national badminton players with a mean age of $15.2 \pm 0.18$ years, length of $170.5 \pm 2.13 \mathrm{~cm}$, body weight of $59.5 \pm 2.48 \mathrm{~kg}$ and sports age of $5 \pm 1.36$ years participated in 
Orhan, Ö., Çetin, E., Yarım, İ., \& Gültekin, O. (2019). Elit badmintoncuların smaç vuruşu isabet oranları ve sıçrama performansları ilişkisinin değerlendirilmesi. Journal of Human Sciences, 16(3), 815-822. doi:10.14687/jhs.v16i3.5761

the study. Volunteer consent form was signed by the participants and the study was conducted by the Helsinki Declaration Principles. The study was planned for two days and the first day height, body weight (Seca) and leg strength were measured. On the second day, after the Squat Jump (SJ) jump was recorded, the dunk hit protocol was applied. Leg strength test dynamometer (ProsportTMR HBD 1000 dynamometer), jump parameters of the subjects were measured with Opto Jump (Microgate, Bolzano). The smash swere performed as $3 \times 30 \mathrm{sec}$ and 3 minutes passive rest protocol was applied after each series. In every 30 seconds, the shots were made by the national team coach with the multishuttle method as 5-10-15. The hit of the parallel smash scored was recorded by the referee. Data analysis was performed using SPSS 23.0 package program. For the relationship between the parameters, Spearman Correlation test and Friedman test were used for comparison of parameters with intermittent jump. As a result of the study, it was seen that there were statistically significant differences in the power and contact time parameters applied during the jumps as the number of smash increased within 30 seconds. When hit rates, players scored $36 \%$ on 5 smash, $26 \%$ on 10 smash and $23 \%$ on 15 smash. At the end of the firing period, $95.9 \pm 2.25$ beats $/ \mathrm{min}$ in 5 beats, $152.8 \pm 3.5$ beats / $\mathrm{min}$ in 10 beats and $166.4 \pm 1.34$ beats / $\mathrm{min}$ in 15 beats were also measured. In addition, it was found that as the frequency of scored increased, the rate of accuracy and heart rate increased. This can be interpreted as a decrease in the scored of the players due to fatigue as the smash frequency increases. As a result of the evaluation of the relationship between recorded hit rates and smash jump heights, leg strength and 15 throw hit rates, no statistically significant results were obtained. Jump is important in all sports but it is one of the important parameters that affect the success for many techniques in the game in badminton (Azmin et al., 2005). In many studies in which the motor skills of badminton players are examined and evaluated, good sprinting is the most popular criterion for good performance when performing jumping technique. It is effective not only in terms of smash technique but also in the success of many different techniques used in deception skills that are frequently used in the game. In a study he conducted, Shetty examined the relationship between motor skills. According to the findings of the jump and lower extremity strength is related (Shetty, 2002). In the study, no statistically significant result was found when the relationship between leg strength and speed jump and smash rates of badminton players was evaluated. However, as the jump frequency increased, the effect of the players on the jump performance was investigated and it was thought that decreased contact time caused the players to use more force. As a result, it was seen that jump performance of badminton players could be affected by increasing the frequency of smash performance in the game. Increasing the frequency of response time to shorten the contact time and find that the power value increases. In addition to all these jump variables, heart rate is rapidly increasing and the hit rates are decreasing. In summary, it can be said that the quality of bounce performances may have an impact on the match score. 